\title{
Zur Kenntnis des Smegmafettes der Pferde.
}

\section{Von \\ Dr. Franz Zaribnicky.}

(Aus dem Laboratorium für medizinische Chemie an der k. und k. Tierärztlichen Hochschule in Wien.)

(Der Redaktion zugegangen am 10. Juli 1912.)

Die Untersuchung derjenigen Fette, welche durch die Haut abgesondert werden, begegnet großen Schwierigkeiten, welche z. T. auch darin ihren Grund haben, daß ausreichende Mengen von Untersuchungsmaterial nicht leicht zu bekommen sind. Eine Ausnahme bildet nur das Hautfett vom Schafe, welches als Wollfett (Lanolin) in beliebiger Menge zugänglich ist (eingehende Untersuchungen von Darmstaedter und Lifschütz, ${ }^{1}$ ) Marchetti) ${ }^{2}$ ). Beim Menschen behalf man sich damit, daß man das Fett von Dermoidcysten (E. Zdarek $;^{3}$ ) Franz Ameseder $\left.{ }^{4}\right)$ ) und das Fett der vernix caseosa von Neugeborenen (L. Ritter von $\left.\mathrm{Zumbus} \mathrm{ch}^{\mathbf{5}}\right)$ ) untersuchte. Die Gewinnung größerer Mengen dieser beiden Materialien setzt aber immerhin eine mühsame, emsige Sammelarbeit voraus.

Im folgenden will ich auf ein Material aufmerksam machen, welches namentlich in größeren Städten, in denen Pferdeschlachthäuser bestehen, leicht in großer Menge gesammelt werden kann.

Der Präputialsack beim Pferde (Hengst oder Wallach) ist in der Regel mit einer grauschwarzen, äußerst übelriechenden, schmierigen Masse erfüllt. Diese Masse stellt das Smegma der Pferde, das Sekret der glandulae praeputiales dar, vermengt mit Staub, Schmutz, Haaren und Epidermisschollen.

Für die Zwecke der Untersuchung wurde das Smegma den lebenden Pferden mit der zuvor gut gereinigten Hand aus dem praeputium in zusammenhängenden Stücken von wechselnder Größe entnommen und in ein bereit stehendes, trockenes, mit eingeriebenem Stöpsel versehenes Glas gegeben. Von etwa

1) Berichte d. deutsch. chem. Gesellschaft 1896, Bd. 29, S. 618, 1474 u. 2890 und 1898, Bd. 31, S. 97.

2) Ibidem, 1896, Bd. 29, S. 19.

3) Diese Zeitschr., B. 57, S. 461-63.

4) Ibidem, Bd. 52, S. 121-28.

5) Diese Zeitschr., Bd. 59, S. 506-19). 
25-30 Pferden konnte so leicht $1 \mathrm{~kg}$ Smegma gesammelt werden. Dieses kam nun in einen großen Ätherextraktionsapparat nach Soxhlet, wo das ganze Fett mit Äther erschöpfend extrahiert wurde. Nach Verjagen des Äthers und Trocknen des Rückstandes in einem trockenen Strome von Wasserstoffgas unter den üblichen Vorsichtsmaßregeln blieben ca. 85,0 g einer lichtbraunen fettartigen Masse von salbenartiger Konsistenz zurück.

Ich habe dieses Fett so weit untersucht, daß eine gewisse Ähnlichkeit mit dem Fette der Dermoïdcysten und der vernix caseosa konstatiert werden konnte. Diese Untersuchung ergab folgende Resultate:

Säurezahl: $5,4366 \mathrm{~g}$ Fett verbrauchten $119,9 \mathrm{ccm}$ Lauge $(1 \mathrm{ccm}=0,0973 \mathrm{ccm}$ normal); Säurezahl 120,4.

Verseifungszahll I: 1,6056 g Fett verbrauchten $22,4-13,3$ $=9,1 \mathrm{ccm}$ Salzsäure $(1 \mathrm{ccm}=34,87 \mathrm{mg} \mathrm{KOH})$; Verseifungszahl 197,6 .

II: $2,1662 \mathrm{~g}$ Fett verbrauchten $21,8-9,6=12,2 \mathrm{ccm}$ Salzsäure $(1 \mathrm{ccm}=34,87 \mathrm{mg} \mathrm{KOH})$; Verseifungszahl 196,4; Mittel 197,0.

Reichert-Meisslsche Zahl: 5,4366g Fett verbrauchten 2,04 ccm n/10-Lauge; Reichert-Meissl Zahl 1,88.

Jodzahl: $0,5795 \mathrm{~g}$ Fett verbrauchten $31,1-8,5=22,6 \mathrm{ccm}$ Thiosulfatlösung (1 $\mathrm{ccm}=0,01263 \mathrm{~g}$ Jod); Jodzahl : 49,2.

Acetylzahl der Fettsäuren: $2,6337 \mathrm{~g}$ acetylierte Fettsäuren verbrauchten zur Neutralisation $74,3 \mathrm{ccm}$-Lauge (1 $\mathrm{ccm}=0,0973 \mathrm{ccm}$ normal); Acetylsäurezahl 154,0.

2,1098 g acetylierter Fettsäuren verbrauchten nach der Verseifung mit überschüssiger, alkoholischer Kalilauge 21,7-9,5 $=12,2 \mathrm{ccm}$ Salzsäure $(1 \mathrm{ccm}=0,62148 \mathrm{~cm}$ normal $)$; Acetylverseifungszahl 201,5, demnach Acetylzahl 47,5.

Bestimmung des freien und zu Estern gebundenen Cholesterins und des unverseifbaren Rückstandes.

Nach der Methode von A. Windaus ${ }^{1}$ ) verarbeitet, lieferten $3,0957 \mathrm{~g}$ Fett $0,1358 \mathrm{~g}$ Digitonincholesterid aus freiem

1) A. Windaus, Quantitative Bestimmung des Cholesterins und der Cholesterinester,, Diese Zeitschr. Bd. 65, S. 111. 
Cholesterin, entsprechend 0,03301 $\mathrm{g}$ freiem Cholesterin $=1,07 \%$ und $0,4672 \mathrm{~g}$ Digitonincholesterid aus zu Estern gebundenem Cholesterin $=0,11358 \mathrm{~g}$ gebundenem Cholesterin $=3,67 \%$.

Nachdem das freie Cholesterin abgeschieden war, betrug die Menge des unverseifbaren Rückstandes $0,3295 \mathrm{~g}$, demnach samt dem freien Cholesterin 0,3625 $\mathrm{g}=11,71 \%$.

Zur Übersicht seien die Resultate in einer Tabelle zusammengestellt:

$\begin{array}{lc}\text { Säurezahl } & 120,4 \\ \text { Verseifungszahl } & 197,0 \\ \text { Reichert-Meissl-Zahl } & 1,88 \\ \text { Jodzahl } & 49,2 \\ \text { Acetylzahl der Fettsäuren } & 47,5 \\ \text { Unverseifbarer Rückstand } & 10,64 \% \\ \text { Freies Cholesterin } & 1,07 \% \\ \text { Zu Estern geb. Cholesterin } & 3,67 \% \\ \text { Zusammen Cholesterin } & 4,74 \%\end{array}$

Das Cholesterin bildete demnach nur einen Teil des unverseifbaren Rückstandes, nämlich nur 31,33\%. Um nun ungefähr einen Anhaltspunkt für die Natur des übrigen unverseifbaren Rückstandes zu gewinnen, wurden diese Stoffe in größerer Menge dargestellt. Ungefähr $50 \mathrm{~g}$ Fett wurden mit alkoholischer Kalilauge verseift, die verseifte Masse wurde auf dem Wasserbade zur Trockne verdampft, der Abdampfrückstand im warmem Wasser gelöst und die rohen Fettsäuren (samt dem unverseifbaren Rückstande) aus der Lösung durch Salzsäure ausgeschieden. Der mehrfach mit Wasser gewaschene Fettsäurekuchen wurde in warmer verdünnter Sodalösung gelöst und die Lösung mit Chlorcalcium ausgefällt. Der Niederschlag wurde auf einem Filter gesammelt, mit Wasser ausgewaschen, auf dem Wasserbade getrocknet, zerrieben und im Soxhletschen Apparate anhaltend mit Äther extrahiert. Aus dem Verdunstungsrückstande der Ätherlösung wurde in üblicher Weise das Cholesterin durch Digitonin abgeschieden. Die vom Digitonincholesterid abfiltrierte Flüssigkeit lieferte, nachdem das im Überschuß zugesetzte Digitonin entfernt worden war, einen gelblich öligen, zum Teile krystallinischen Abdampfrückstand. 
Dieser Abdampfrückstand enthielt noch Spuren von Fettsäuren, er wurde daher in Äther gelöst und die Lösung wiederholt mit verdünnter Sodalösung ausgeschüttelt. Der Äther hinterließ nunmehr 0,8 g eines weißlichen, öligen, amorphen Rückstandes, welcher gegen Phenolphthalein nicht die leiseste Spur einer sauren Reaktion mehr zeigte.

Dieser Verdunstungșrückstand wurde in Eisessig gelöst und die Lösung so lange mit einer Auflösung von Chromsäure in Eisessig versetzt, bis sie einen bräunlichen Farbenton beibehielt. Dann wurde in Wasser gegossen und das Ausgeschiedene durch wiederholtes Umschmelzen in heißem Wasser von Chromverbindungen befreit.

Das so gereinigte Oxydationsprodukt bildete eine farblose, krystallinische, sauer reagierende Substanz.

0,2611 $\mathrm{g}$ des Oxydationsproduktes, in einem Gemenge von Alkohol und Äther gelöst, verbrauchten zur Neutralisation gegen Phenolphthalein 4,2 ccm Lauge $(1 \mathrm{ccm}=0,1066 \mathrm{ccm}$ Normal). Unter der Voraussetzung, daß das Oxydationsprodukt eine einheitliche Säure wäre, würde dieser Versuch für das Oxydationsprodukt ein Äquivalentgewicht von 583 ergeben.

Die neutralisierte Flüssigkeit wurde mit Wasser verdünnt und mit ein wenig mehr. Silbernitratlösung versetzt, als sich aus dem vorhergehenden Versuche berechnete, nachdem der Versuch einer fraktionierten Fällung mit Silbernitratlösung fehlgeschlagen war. Der entstandene Niederschlag wurde auf einem Filter gesammelt, mit Wasser ausgewaschen und getrocknet. $0,1633 \mathrm{~g}$ des trockenen Niederschlages hinterließen beim Glühen 0,0314 $\mathrm{g}$ Silber, unter der Annahme einer einheitlichen Substanz entsprechend einem Äquivalentgewichte von 454 .

Überblickt man diese Untersuchungsergebnisse, so springt zunächst der Reichtum des Pferdesmegmafettes an höheren Alkoholen in die Augen. In dieser Hinsicht stimmt dieses Fett mit anderen Hautfetten überein. Von diesen höheren Alkoholen ist ein relativ zwar geringer, an sich aber beträchtlicher Teil Cholesterin, welches zum Teile in freiem Zustande im Fett enthalten ist, zum andern und zwar größeren Teile in Form von Estern mit Fettsäuren.

Die Versuche, welche ich angestellt habe, um einiges 
über die anderen Alkohole zu erfahren, bezweckten keineswegs eine völlige Erkenntnis dieser Alkohole, sie sollten vielmehr nur einer allgemeinen Orientierung dienen. Aus den Versuchen geht nun zweifellos hervor, daß unter diesen höheren Alkoholen sich primäre finden, weil sie durch Chromsäure zu Säuren oxydiert wurden. Das hohe Äquivalentgewicht, welches gefunden worden ist, dürfte indessen kaum auf ein hohes Molekulargewicht der Alkohole $\mathrm{zu}$ beziehen sein. Es ist vielmehr zu vermuten, daß das gefundene hohe Äquivalentgewicht der Säuren auf einer Täuschung beruht, indem den Säuren noch andere Stoffe von nicht saurer Natur beigemengt waren. Daraus kann vermutet werden, daß unter den höheren Alkoholen außer Cholesterin und außer primären Alkoholen noch andere, sekundäre oder tertiäre, Alkohole enthalten sind, welche durch Chromsäure nicht zu Säuren oxydiert werden konnten.

Von den in dem Fette enthaltenen Fettsäuren ist ein großer Teil frei, wie es die Säurezahl ausdrückt. Es darf dies indes nicht wundernehmen, weil das Smegma in ständiger Zersetzung begriffen ist. Es darf wohl auch die Menge der flüchtigen Fettsäuren größer angenommen werden, als durch die Reichert-Meisslsche Zahl ausgedrückt wird, indem gewiß ein großer Teil der freien, flüchtigen Säuren bei der Abscheidung und Trocknung des Fettes verloren gegangen ist.

Die gefundene Jodzahl ist weit größer, als daß sie nur durch das Cholesterin erklärt werden könnte; es muß demnach noch eine beträchtliche Menge ungesättigter Säuren in dem Fette enthalten sein. Ferner darf auch die Anwesenheit von Oxysäuren vermutet werden. Rechnet man nämlich den ganzen unverseif baren Rückstand als Cholesterin, so würden dadurch nur etwa $3 / 8$, rechnet man ihn aber als Cetylalkohol, so würde dadurch etwas mehr als die Hälfte der Acetylzahl bedeckt; der Rest der Acetylzahl käme auf Oxysäuren. Im übrigen berechnet sich aus den vorliegenden Daten für die Fettsäuren ein durchschnittliches Molekulargewiçht, welches dem der Palmitinsäure nahesteht. Darnach dürften bei einer eingehenden Untersuchung der Fettsäuren des Smegmafettes keine auffallenden Resultate zu erwarten sein. 